\title{
LOS VALORES DE LA CASA-ESTUDIO DE LUIS BARRAGÁN
}

\author{
Cruz López Viso
}

Boletín Académico. Revista de investigación y arquitectura contemporánea

Escuela Técnica Superior de Arquitectura. Universidade da Coruña

elSSN 2173-6723

unw.boletinacademico.com

Número 1 (2011)

Páginas 09-17

Fecha de recepción $\quad 20.10 .2010$

Fecha de aceptación $\quad 17.01 .2010$

https://doi.org/10.17979/bac.2011.1.0.958

\section{Resumen}

El siguiente texto es una síntesis de los valores que se extraen de una obra del arquitecto Luís Barragán, su casa construida en la colonia de Tacubaya en México DF el año 1947. Mediante las herramientas de análisis se construye un discurso analógico que esclarece la obra.

\section{Abstract}

The following text is a summary of the conclusions reached during the research of the 1947 Luís Barragán's house built in Tacubaya (Mexico DF). The concepts and tools of my analysis approach Architecture in such a way that it reveals its values as well as the references that connect it to other places.

\section{Palabras clave}

Barragán. Percepción. Hapticidad. Límite. Experiencia.

\section{Keywords}

Barragán. Perception. Hapticity. Limit. Experience. 


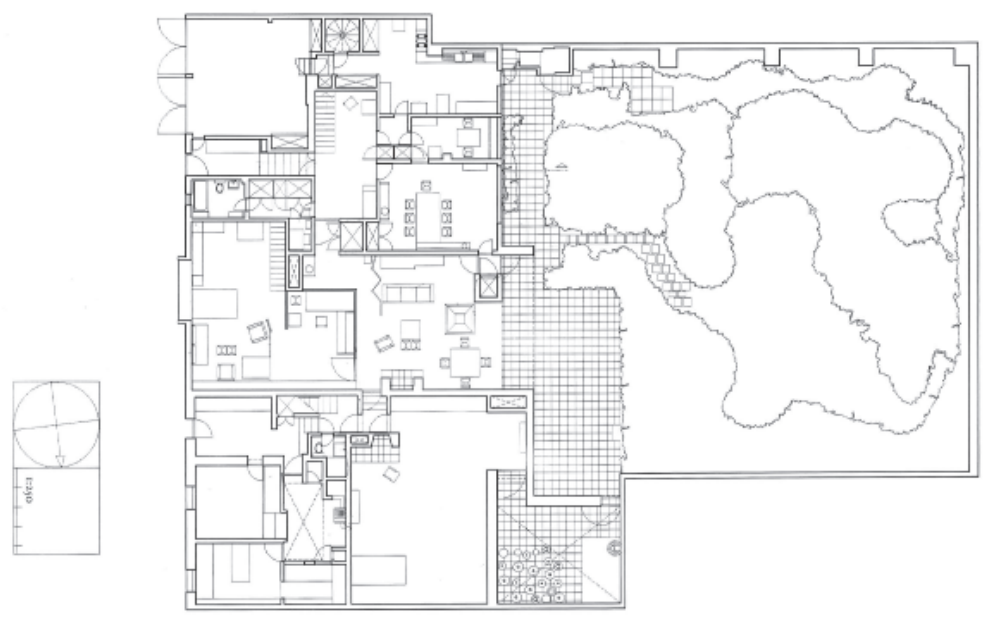

1 Luis Barragán, casa Barragán (Ciudad de México, 1947/48); planta baja.

\section{CASA Y ESTUDIO DE LUIS BARRAGÁN}

En 1947 Luis Barragán (1902/88) construye su casa y estudio en la colonia de Tacubaya en México DF (lugar en el que vive desde 1948 hasta su muerte en 1988). Esta obra inicia su etapa de madurez. En ella confluyen valores que ha elaborado desde sus primeras búsquedas. Hasta llegar a 1947 hay un proceso conectado con lo que sucede en el contexto mexicano y las influencias que el arquitecto recoge en sus viajes. Su obra no está completamente definida. A partir de la casa-estudio los fundamentos de su arquitectura son estables.

«La casa-estudio representa un laboratorio permanente de soluciones y modificaciones imperceptibles de órdenes y equilibrios buscados durante largo tiempo» ${ }^{1}$.

En 1939 adquiere el terreno en la colonia de Tacubaya (4000 m2.) «para realizar tres jardines, dividiéndolo posteriormente para construir dos viviendas. Una fue vendida al cabo de unos pocos ańos, la casa Ortega, y en la otra Barragán construye y fija su residencia personal. A través de esta operación de segregación realiza dos proyectos de viviendas en diferente tiempo, exentos de formalismos de cara al exterior que se integran en el contexto humilde que a finales de los años 40 se había desarrollado en esa zona. En la búsqueda obstinada de un nuevo orden que diese sentido a las innumerables imágenes acumuladas en la memoria y a los diversos intentos experimentales de la década anterior, iniciaba así la época de madurez»².

\section{ETAPAS EN LA OBRA DE BARRAGÁN}

Luis Barragán vive su infancia en Jalisco, donde están ubicadas sus primeras obras cuyo origen se encuentra en la arquitectura vernácula tapatía. En 1924 realiza su primer viaje a Europa, que lo acerca a la obra de Ferdinand Bac. Al mismo tiempo, en 1925, dentro de la Exposición Universal de París, Le Corbusier construye el Pabellón de l'Esprit Nouveau —un auténtico manifiesto de la nueva arquitectura-, donde la naturaleza se enmarca de un modo sofisticado y abstracto. Barragán se mueve entre estos dos polos: la oscilación entre la mirada romántica (Bac) y el magnetismo que ejerce Le Corbusier. En 1931 (su segundo viaje a Europa), conoce personalmente a Ferdinand Bac y parece confirmarse cierta afinidad entre ellos. Conoce también a Le Corbusier, que desde su madurez (tiene 44 años) estimula a un Barragán de 29. Desde su retorno (1931/35), Barragán seguirá su actividad en Guadalajara, y en 1935 se traslada a México DF, donde adopta las formas del racionalismo europeo para retomar posteriormente el origen de su búsqueda en las primeras casas de Guadalajara: la casa Luna (1928) y la casa G. Cristo (1929). Después de unos años de intensa actividad (1936/40) se detiene, y durante cuatro años (1940/44) experimenta en la casa Ortega (su primera vivienda colindante con la casa-estudio) para acometer con lo aprendido la construcción de su casa definitiva. El arquitecto, tras un proceso de experimentación, consolida su nueva directriz creativa en la casa de Tacubaya (1947/48) (Fig. 01). Muere en 1988. 

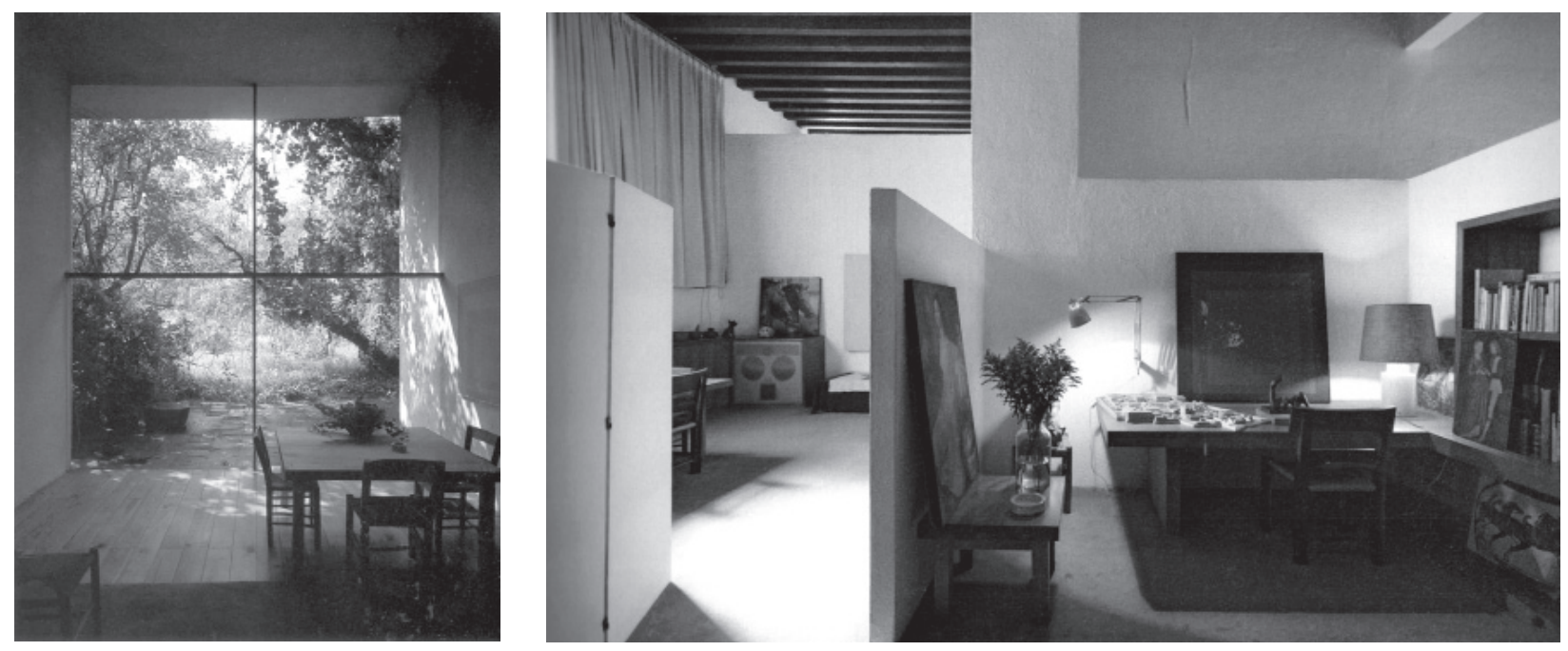

2 Casa Barragán; la estancia.

3 Casa Barragán; la biblioteca vista desde la estancia.

\section{HERRAMIENTAS DE ANÁLISIS. CONCEPTOS}

PERCEPCIÓN. La percepción es un proceso cognitivo de carácter inferencial (creativo y constructivo) en el que los estímulos desde los sentidos son interpretados y activados por la memoria. Voy a citar unas palabras de Jonathan Crary (catedrático de Historia del Arte en la Universidad de Columbia), que se encuentran en su obra Suspensiones de la percepción:

Al privilegiar la categoría de lo visual se corre el riesgo de ignorar las fuerzas de especialización que dieron lugar a esta categoría para convertirla en el concepto intelectual que manejamos hoy. Lo visual puede desviarse hacia modelos de percepción y subjetividad que se alejan de la idea de corporeidad (...) El uso que hago del problemático término percepción es una manera de aludir a un sujeto que se define más allá del sentido único de la visión, en términos que incluyen al oído, al tacto y lo que es más importante a modalidades sensoriales que por lo general reciben poca o ninguna atención en los estudios visuales. ${ }^{3}$

Las raices de la palabra atención resuenan con el sentido de tensión, de estar estirado y también de esperar. Sugieren la posibilidad de fijación, de mantener la fascinación o contemplación hacia algo, en la que el sujeto que observa está simultáneamente inmóvil y desanclado. Pero al mismo tiempo, una suspensión es también una cancelación o interrupción, y quería aludir a una interrupción, o incluso a una negación de la percepción misma. ${ }^{4}$
A partir de este umbral de atención surge el concepto de mirada envolvente y la percepción focalizada en el ojo se transforma en otra que integra todos los sentidos. El crítico de arte Norman Bryson se refiere al valor de la «mirada de otra visión envolvente que se desvanece por todos lados» ${ }^{5}$. La mirada en campo extendido. Desde esta aproximación se transforma lo visible en táctil.

HAPTICIDAD. La voz háptica (que no aparece en el diccionario de la Real Academia Española) viene del griego haptô (verbo-tocar) y haptikós (adjetivo-táctil), relativo a las sensaciones que percibimos a través de la piel, de la estructura muscular y la estructura ósea. Juhani Pallasmaa en Los ojos de la piel hace mención a una arquitectura háptica que estimula percepciones no visuales. La hapticidad enfatiza la cualidad táctil del espacio, destacando la materialidad y textura frente a la forma. "Frente a la realidad hiperconstruída que revela el ojo es preciso sensibilizar la arquitectura mediante un sentido fortalecido de materialidad, hapticidad, textura, peso, densidad del espacio y luz materializada ${ }^{6}$.

EXPERIENCIA. El concepto de experiencia directa fue desarrollado por el maestro Nishida (1870-1945) de la Escuela de Kyoto (Kitaro Nishida, Hajime Tanabe y Keiji Nishitani). La Escuela de Kyoto se funda en 1932 dentro de la Universidad Imperial a partir de las reflexiones de Nishida, catedrático de filosofía, y es paradigma de una reflexión intercultural. La experiencia directa es anterior al análisis intelectual: es el asombro, el sentirse siendo, la unidad del yo con el mundo, la 


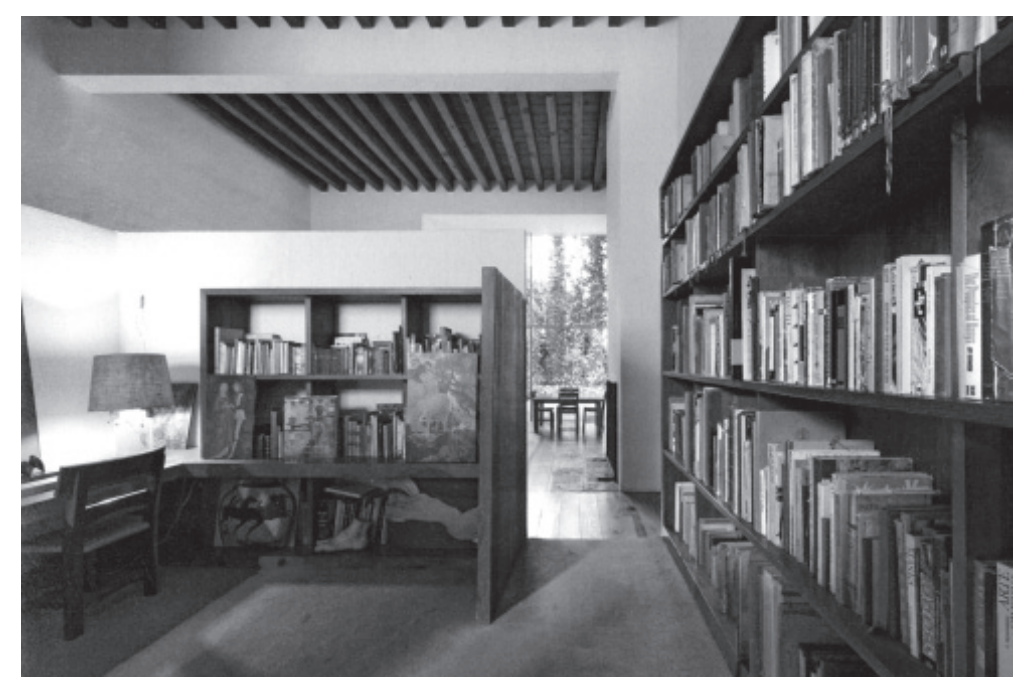

4 Casa Barragán; la biblioteca.

disolución de sujeto y objeto. El conocimiento sin juicios ni evaluación, donde los límites desaparecen.

LÍMITE. Partiendo de la idea de límite empleada por los romanos — «limes, franja estrecha y oscilante, o movediza, pero habitable y susceptible de colonización, cultivo y culto» ${ }^{7}$ - el concepto de límite aparece asociado a la idea de movilidad. No se trata de un espacio estable, sino de una epidermis viva y cambiante. El límite rompe la continuidad de un campo determinado, pero para que el propio límite como discontinuidad desaparezca, primero debe existir y fundirse en el campo total de la percepción. Existen dos espacios fundamentales, dos $\operatorname{cercos}^{8} \mathrm{y}$ un límite que los une y escinde, y que es tanto frontera en su sentido de definición de un lugar como apertura.

LÍMITE FENOMENOLÓGICO. Un límite fenomenológico se crea desde las fluctuaciones de fenómenos naturales. No es una línea física sino un ámbito oscilante, una zona sensible a la luz, al sonido, a la temperatura... un lugar donde experimentamos un cambio. El lugar emerge de la acción de desenfocar lo percibido cuando el límite desaparece. Determinadas obras de Barragán contienen umbrales donde se produce un «movimiento oscilante entre los límites sensibles»?.

ATMÓSFERA. El concepto de atmósfera está ligado a lo que Peter Zumthor denomina calidad arquitectónica ${ }^{10}$, y que, según él, se obtiene prestando atención a determinados parámetros que construyen los límites: la armonía de los materiales, la temperatura del espacio, la tensión entre interior y exterior, los grados de intimidad, la coherencia, la belleza, la arquitectura como entorno, los objetos, la magia de lo real (el espesor del tiempo, la capacidad de un lugar de evocar otros lugares) o la anatomía de lo arquitectónico...

El límite más evidente en la arquitectura de Barragán es el muro, que se manifiesta en una primera aproximación como elemento que separa dominios de diferente naturaleza: interior-exterior, cielo-tierra... poniendo énfasis en la polaridad. Experimentando la casa se hace una exploración de los parámetros que construyen los límites mediante las herramientas que se proponen. Una arquitectura de cualidades físicas rotundas - peso, textura, geometría - cuya materialidad, al interceptar con los sentidos, pierde consistencia. ¿Por qué cerrando los ojos, el sonido de un avión no ensombrece el sonido del agua? ¿Por qué la caída de las hojas, su fermentación, se convertía en un signo de algo más? Estos movimientos de la naturaleza en cualquier otro lugar serían invisibles o insignificantes. ¿Por qué en esta casa eran trascendentes?

¿Qué entendemos por ámbito? El ámbito no es aquello donde las cosas se alojan espacialmente. El ámbito no es un espacio; es pura y simplemente una espaciosidad. $Y$ el ámbito no es espacio absoluto en ningún sentido (...) es algo pre-espacial, lo que hace posible que haya espacio. ${ }^{11}$

La arquitectura tiene su propio ámbito existencial. Dado que mantiene una relación especialmente corporal con la vida, no es ni mensaje ni signo, sino una 
cobertura y un trasfondo de la vida que junto a ella transcurre... ${ }^{12}$

Estamos dentro mirando hacia el jardín. Ambos lugares se enlazan creando un ámbito que no permite el movimiento, ya que el paso se produce desde una puerta lateral (una esclusa). Esa imposibilidad de traspasar el límite, adentra el jardín mediante la transparencia, y aunque ese límite es hermético, se desarrollan extensiones táctiles. La casa se recoge en sí misma y entreabre espacios que la amplían (Fig. 02).

\section{HERRAMIENTAS DE ANÁLISIS. AFINIDADES}

LA CASA JAPONESA. Pueden establecerse afinidades entre la cultura del límite en Japón y el entendimiento que hace Barragán de la definición de los lugares, de cómo nos encontramos en un área de la casa sin saber cuando llegamos a ella, porque ese lugar se separa de otro mediante límites que se transforman. El habitante experimenta gradualmente una sucesión de áreas definidas por elementos móviles donde los lugares tienen funciones, pero no estructuras. En el japonés antiguo, tiempo y espacio se conciben como intervalos. Existe un concepto que integra espacio y tiempo, ma. Significa pausa, vacío potencialmente cargado para cobrar conciencia de nuestra subjetividad por medio del propio cuerpo.

Afinidades existentes entre la casa japonesa y la casaestudio son: el empleo de los materiales en su naturaleza original con el desgaste (sabi), la pátina y las imperfecciones (wabi), el uso sutil de luces y sombras para marcar la atmósfera de los lugares - a diferencia de Occidente- donde la casa se abre a la luz, la articulación de cavidades mediante umbrales para fundirse en un solo espacio, la fachada simplificada al máximo con un hueco de entrada que pasa desapercibido y no invita al acceso, el entendimiento de la naturaleza desde el marco interior de la casa, el orden partiendo del ángulo recto solapando unos espacios con otros como umbrales enlazados, la sofisticación desde la simplicidad y el poder simbólico que adquieren las formas al reducirse el número de elementos que intensifican su fuerza expresiva.

TOKO NO MA. La penumbra es la zona ambigua donde se confunden sombra y luz. Cuando Barragán explica la importancia de los muros para recoger los lugares y acotarlos se refiere «a la necesidad humana - como mamíferos que somos- de penumbra. Necesitamos un refugio... incluso en contra de la luz» ${ }^{13}$.

Dentro de la casa japonesa se crea un lugar - toko no ma - para intensificar la profundidad de la penumbra. Ahí se guarda el ídolo que irradia luz. En la casa de Tacubaya se crean recodos donde la luz llega modulada por múltiples deslizamientos y se condensa creando reflejos sorprendentes. Del mismo modo que en la casa tradicional japonesa el buda se cubre con oro laminado para que dentro de la oscuridad la mínima luz cristalice, mediante los lienzos de Mathías Goeritz el arquitecto utiliza el dorado para refractar la luz.

El envejecimiento de las cosas, el marchitarse de las 


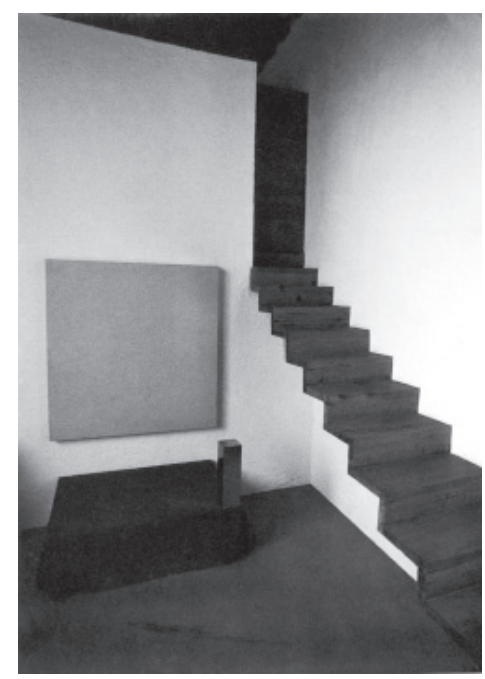

5 Casa Barragán; la escalera de la biblioteca.

flores, el fluctuar del pensamiento, las sombras cayendo sobre el agua o sobre la tierra son los tipos de fenómenos que más profundamente impresionan al japonés. El gusto por el movimiento en estos fenómenos impregna la concepción japonesa del espacio arquitectónico en el que, por ejemplo, un paramento de tablas puede parecer transparente al permitir la penetración de la luz y de lineas de visión. Se percibe en este espacio la fluctuación de las sombras, un momentáneo cambio entre los mundos de la realidady de la irrealidad. Ma es el vacio momento de espera para este cambio. ${ }^{14}$

LUZ. La realidad física de México DF es esencial para entender las obras de Barragán. El sol omnipresente determina la gradación de movimientos hacia la luz que en la casa-estudio es matizada mediante filtros que funcionan como superficies refractarias. Las condiciones topográficas de la ciudad, su altitud, la extensión y su intensidad luminosa determinan la creación de dispositivos que amortigüen el tránsito entre el espacio privado y el espacio urbano. La luz se adentra en Tacubaya suavizada. Es un elemento elaborado. Dentro de la casa, establece vínculos con su contexto, otorgando al lugar interior la medida del tiempo, los ciclos y las estaciones. En la casa la materia no está atirantada, pero existe resistencia a la fuente de luz. Barragán emplea los huecos en diferentes formatos, cuya luz es graduada por contraventanas articuladas empleando veladuras, cortinas o celosías. Respecto al jardín, las ramas entrelazadas crean una bóveda natural cuya filigrana móvil la refleja y transparenta hacia el interior.

Es preciso diferenciar calidad de cantidad de luz. Para obtener luz de calidad se requiere poner atención no solo a la ubicación de los huecos y su tamaño, sino a todos los sistemas que la regulen y modelen para resaltar la plasticidad de los muros y el relieve de sus texturas. El otro recurso empleado es no llevar los muros y tabiques hasta el techo cerrando las cavidades. En la planta baja, los lugares se organizan mediante compartimentos adheridos a espacios mayores (Fig. 03 y 04 ), semiabiertos y permeables; por ejemplo el tapanco (la celda dentro de la casa), lugar de reserva y recogimiento abierto a la biblioteca (Fig. 05).

\section{AGUAS.}

En los Vedas, las aguas reciben el apelativo de mâtritamâh (las más maternas), pues al principio todo era como un mar sin luz. En general, en la India se considera a este elemento como el mantenedor de la vida (...) Las aguas simbolizan la unión universal de virtualidades, fons et origo, que se hallan en la precedencia de toda forma o creación. La inmersión en la aguas significa el retorno a lo preformal, con su doble sentido de muerte y disolución, pero también de renacimiento y nueva circulación, pues la inmersión multiplica el potencial de la vida (...) el agua es el elemento que mejor aparece como transitorio entre el fuego y el aire de un lado -etéreos-y la solidez de la tierra. Por analogía mediador entre la vida y la muerte, en la doble corriente positiva, de creación y destrucción. ${ }^{15}$

De la memoria de los lugares de la hacienda de Corrales, el agua es un elemento que el arquitecto utiliza de diferentes maneras: contenedores primitivos alma- 

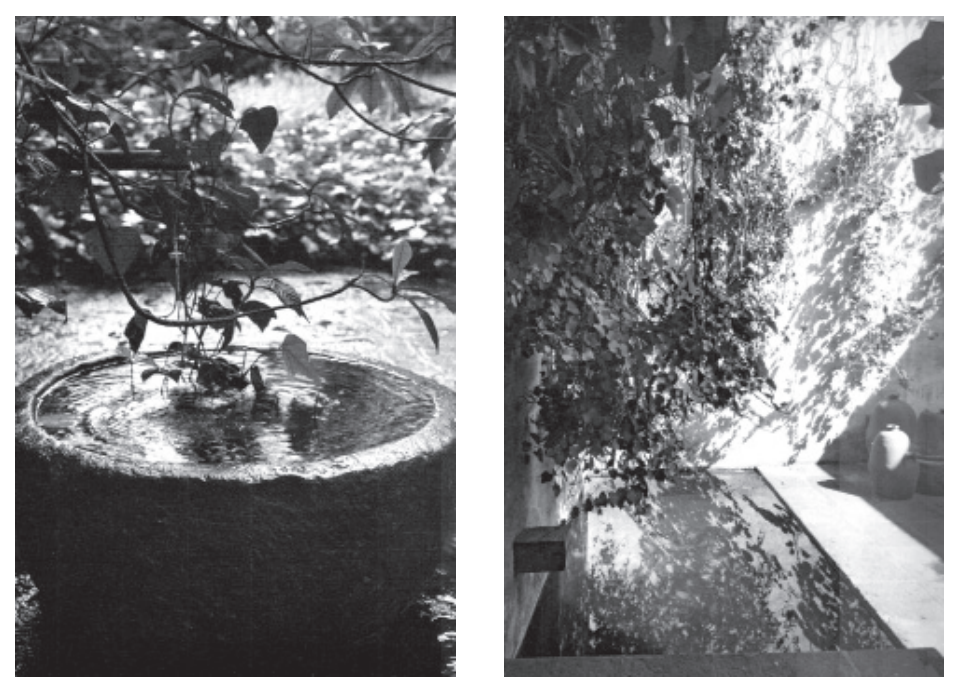

6 y 7 Casa Barragán; izquierda, fuente del jardín; derecha, Patio de las Ollas.

cenan la lluvia dentro de los jardines, bebederos en los espacios abiertos y láminas tan sofisticadas como la que proyecta para la casa Gilardi en el interior de la vivienda. El agua es tectónica como los muros. Puede hacerse un recorrido a través de sus obras para experimentar estas cualidades y su repercusión en el espacio habitado. Desde las micronaturalezas, como el Patio de las Ollas en su casa en Tacubaya, hasta láminas evanescentes como en la casa Gálvez o la sofisticación de la casa Gilardi. El agua como lente fenomenológica incorpora, además de sus recursos plásticos, la dimensión del tiempo (Fig. 06 y 07).

COLOR. Barragán interpreta el color como instrumento que interactúa con la luz y el agua desde el soporte de los muros. Usa dos elementos cromáticos: un color base y otro que emerge detrás. El color aparece en las estancias como una fuerza que asigna orden al espacio y lo focaliza, actuando como límite de los lugares. La atmósfera se crea desde el color-luz que se transforma en luz coloreada complementaria de la interior (Fig. 08).

Creo que, en general, las habitaciones o patios pintados tienen la propiedad de implicar un sol exterior, de captar la luz de afuera. Por tanto, desde un interior $o$ desde un patio entre muros se evoca, se condiciona o se supone una luz que los trasciende: se determina lo que ellos no son. El color produce el efecto de una penumbra coloreada que reclama un sol de luminosidad complementaria. ${ }^{16}$
RUINAS. Las ruinas son la transformación de la materia que se somete al tiempo, la pátina (el efecto del tiempo actuando sobre lo construido). El espesor del tiempo. Ese agotamiento del material es un valor presente en la casi totalidad de la obra de Barragán. Las ruinas son el lugar donde la ausencia sobrepasa en intensidad a la presencia. La obra habla del tiempo vinculado al lugar. Transmite el espesor del tiempo que media entre la primera percepción y la actual. La arquitectura, en esta dimensión, es una entrada al tiempo como ámbito a través del espacio. Y ese campo temporal contiene en su relieve todos los estratos acumulados. Esta idea es esencial en su obra: la transformación del tiempo que es también la transformación de la percepción del espacio.

La contemplación de las ruinas nos permite entrever fugazmente la existencia de un tiempo que no es el tiempo del que hablan los manuales de historia o del que tratan de resucitar las restauraciones. Es un tiempo puro, al que no puede asignarse fecha, que no está presente en nuestro mundo de imágenes, simulacros y reconstituciones, que no se ubica en nuestro mundo violento, un mundo cuyos cascotes, faltos de tiempo, no logran ya convertirse en ruinas. Es un tiempo perdido cuya recuperación compete al arte. ${ }^{17}$

\section{CONCLUSIONES}

Una serie de cuestiones resultan de este acercamiento fenomenológico. ¿Qué límites definen la obra de Barragán? ¿Cómo se materializan? ¿Qué hace que esos límites fluctúen? 


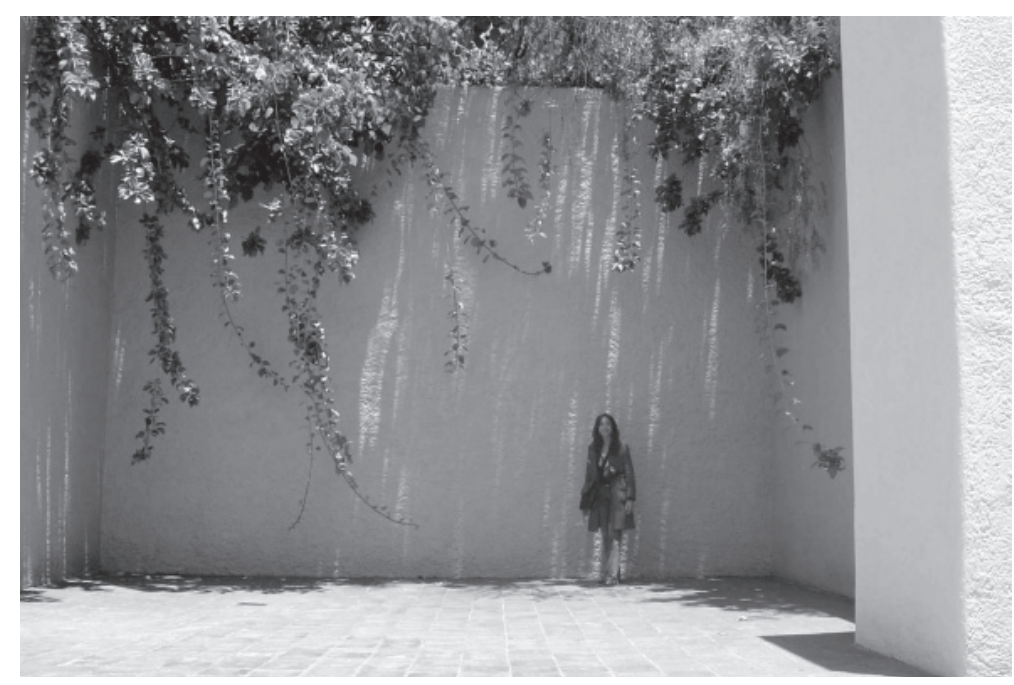

8 Casa Barragán; terraza.

El muro es el elemento esencial que configura esta obra (en el jardín existen contrafuertes que alcanzan los ocho metros de altura, en la azotea los muros van de los tres a los cinco metros). En una primera aproximación, densifica e intensifica el espacio; sin embargo, una vez dentro, los umbrales sensitivos disuelven esa primera percepción de materialidad monolítica para manifestar cualidades más sutiles.

Barragán valora la materialidad sobre la forma, acercándose a los límites que imponen los elementos constructivos (maderas macizas, cueros, pieles, telas, piedras volcánicas...) y evitando el empleo de materiales industriales que desde su ambigüedad restarían intensidad a sus obras. Emplea el menor número de recursos agotando sus posibilidades, y recurre a materiales autóctonos y fórmulas tradicionales destiladas hasta el refinamiento.

El resultado es una obra matérica, que para ser experimentada, precisa de una serie de herramientas que nos permitan trascender los límites que inicialmente percibimos para sentir la atmósfera creada por el arquitecto. 


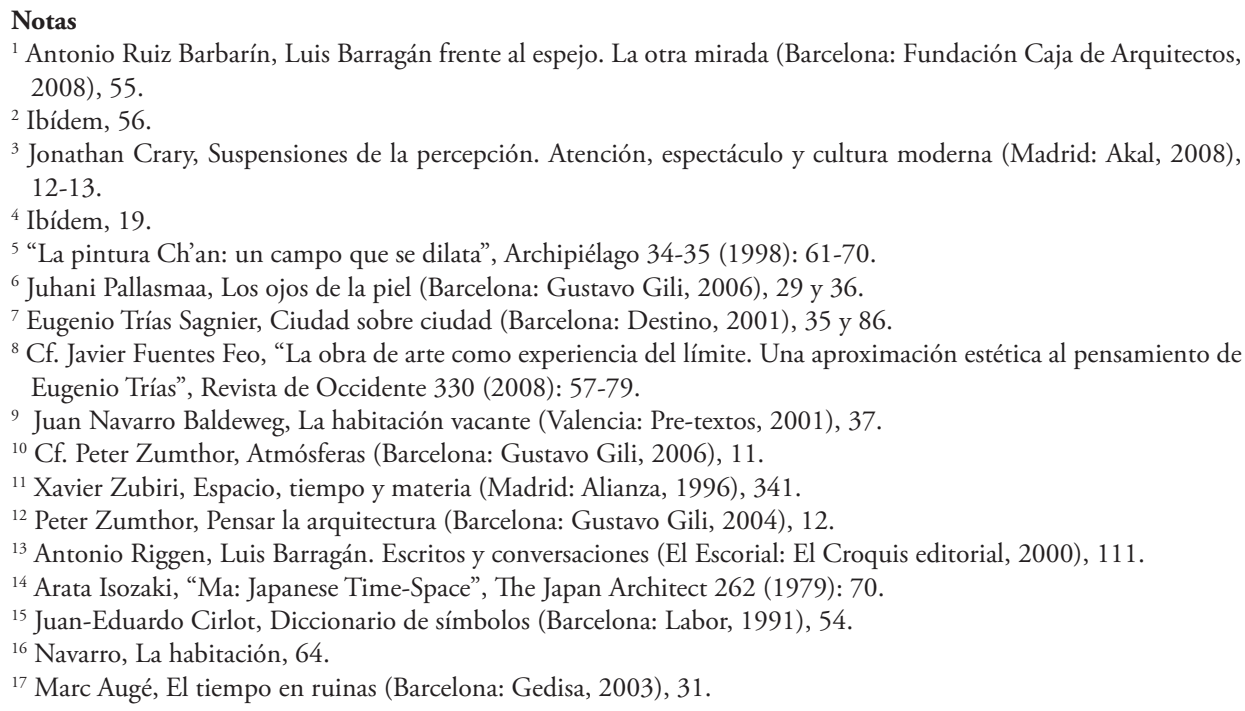

${ }^{3}$ Jonathan Crary, Suspensiones de la percepción. Atención, espectáculo y cultura moderna (Madrid: Akal, 2008), 12-13.

${ }^{4}$ Ibídem, 19

5 “La pintura Ch'an: un campo que se dilata”, Archipiélago 34-35 (1998): 61-70.

${ }^{6}$ Juhani Pallasmaa, Los ojos de la piel (Barcelona: Gustavo Gili, 2006), 29 y 36.

${ }^{7}$ Eugenio Trías Sagnier, Ciudad sobre ciudad (Barcelona: Destino, 2001), 35 y 86.

${ }^{8}$ Cf. Javier Fuentes Feo, "La obra de arte como experiencia del límite. Una aproximación estética al pensamiento de Eugenio Trías”, Revista de Occidente 330 (2008): 57-79.

9 Juan Navarro Baldeweg, La habitación vacante (Valencia: Pre-textos, 2001), 37.

${ }^{10}$ Cf. Peter Zumthor, Atmósferas (Barcelona: Gustavo Gili, 2006), 11.

${ }^{11}$ Xavier Zubiri, Espacio, tiempo y materia (Madrid: Alianza, 1996), 341.

${ }^{12}$ Peter Zumthor, Pensar la arquitectura (Barcelona: Gustavo Gili, 2004), 12.

${ }^{13}$ Antonio Riggen, Luis Barragán. Escritos y conversaciones (El Escorial: El Croquis editorial, 2000), 111.

${ }^{14}$ Arata Isozaki, "Ma: Japanese Time-Space", The Japan Architect 262 (1979): 70.

${ }^{15}$ Juan-Eduardo Cirlot, Diccionario de símbolos (Barcelona: Labor, 1991), 54.

${ }^{16}$ Navarro, La habitación, 64.

${ }^{17}$ Marc Augé, El tiempo en ruinas (Barcelona: Gedisa, 2003), 31.

\section{Procedencia de las ilustraciones}

Fig. 01. Alcérreca, Víctor. Guía Casa Luis Barragán. México DF: Fundación de Arquitectura Tapatía Luis Barragán/ Barragán Foundation, 2004.

Fig. 02. Zanco, Federica. Luís Barragán. La revolución callada. Barcelona: Skira, 2001.

Fig. 03-07. Futagawa, Yukio. Barragán, Luis. Barragán House. México City, México 1947-1948. Tokyo: ADA, 2009. Fig. 08. Fotografía de la autora, 2007.

Sobre la autora

Cruz López Viso. Arquitecta por la ETSAM (1990) y Doctora en Arquitectura desde octubre 2010. Exploradora de campos diversos: pintura, fotografía y arquitectura.

cruzlopezviso@gmail.com 